\title{
Effects of quadratic drag and throughflow on double diffusive convection in a porous layer is
}

\author{
I.S. Shivakumara $^{\mathrm{a}, *}$, C.E. Nanjundappa ${ }^{\mathrm{b}}$ \\ ${ }^{a}$ UGC-Centre for Advanced Studies in Fluid Mechanics, Department of Mathematics, Bangalore University, Bangalore 560001, India \\ ${ }^{\mathrm{b}}$ Dr. Ambedkar Institute of Technology, Department of Mathematics, Bangalore 560056, India
}

Available online 1 December 2005

\begin{abstract}
The linear stability theory is used to investigate analytically the effects of quadratic drag and vertical throughflow on double diffusive convection in a horizontal porous layer using the Forchheimer-extended Darcy equation. The boundaries of the porous layer are considered to be either impermeable or porous, but perfect conductors of heat and solute concentration. Conditions for the occurrence of stationary and oscillatory convection are obtained using the Rayleigh-Ritz method. Stability boundaries are drawn in the Rayleigh numbers plane and the throughflow is found to influence the mode of instability. It is found that, irrespective of the nature of boundaries, a small amount of throughflow in either of its direction destabilizes the system; a result which is in contrast to the single component system.
\end{abstract}

(C) 2005 Elsevier Ltd. All rights reserved.

Keywords: Porous medium; Quadratic drag; Throughflow; Double diffusive convection

\section{Introduction}

Double diffusive convection in porous media has constituted a pole of attraction to researchers because of its applications in varied fields such as geophysics, enhanced recovery of petroleum reservoirs, underground spreading of chemical wastes, sea bed hydrodynamics, crystal growth among others. Copious literature is available on this topic and it is well documented in the book by Nield and Bejan [1]. In the porous layer, the in-situ processing of energy resources such as coal, oil shale or geothermal energy and also packed bed reactors often involves the non-isothermal flow of fluids through porous media, called throughflow. In view of this, the effect of vertical throughflow on convective instability in a porous layer has attracted considerable interest in the recent past, but majority of the investigations have dealt with only single component system [2-5].

Nevertheless, in the kind of problems cited above and also in the directional solidification of concentrated alloys as well as in some energy storage devices, there exist solute concentration gradients in addition to temperature gradients and throughflow in the porous medium. In such situations, the importance of double diffusive instability with

\footnotetext{
th Communicated by A.R. Balakrishnan and S. Jayanti.

* Corresponding author.

E-mail address: isshivakumara@hotmail.com (I.S. Shivakumara).
} 
throughflow becomes significant when precise processing is required. This type of instability problem also has a very strong practical background in geophysics and geo-environmental engineering. The difficulty in dealing with such instability problems is that one has to solve time dependent equations with variable coefficients, and the work in this direction is very limited. Recently, Shivakumara and Khalili [6] have discussed the effect of throughflow on double diffusive convection in a porous layer by considering the Darcy model in which the inertial term is neglected. Also, the boundaries were considered to be adiabatic to both temperature and solute concentration perturbations. However, in the presence of strong throughflow the inertial effects become significant and the Darcy law which holds for small velocities doesn't take care of these effects. Under the circumstances, the use of a non-Darcian model is warranted for a better understanding of the problem. For many naturally occurring porous media the appropriate inertia term in the momentum equation is $|\vec{q}| \vec{q}$ which is a drag term quadratic in the velocity $\vec{q}$.

The main aim of this paper is, therefore, to investigate the combined effects of quadratic drag and vertical throughflow on double diffusive convection in a porous layer by employing the Forchheimer-extended Darcy equation. In the present study, the boundaries of the porous layer are assumed to be perfect conductors of heat and solute concentration which are either porous (i.e. maintained at constant pressure) or impermeable (i.e., maintained at constant velocity and hence impermeable to velocity perturbations). Analytical expressions for the thermal Rayleigh number for the occurrence of both steady and oscillatory onset are obtained for four different types of velocity boundary conditions using Rayleigh-Ritz's method.

\section{The eigenvalue problem and its solution}

We consider a horizontal binary fluid saturated porous layer of thickness $d$ with a constant vertical throughflow of magnitude $w_{0}$ which is either gravity aligned or antigravity in its direction. A Cartesian coordinate system $(x, y, z)$ is chosen such that the origin is at the bottom of the layer and $z$-axis is vertically upward. The boundaries of the porous layer are kept at constant but different temperatures and solute concentrations. That is, $T_{0}$ and $S_{0}$ at the lower boundary $z=0$ while $T_{1}\left(<T_{0}\right)$ and $S_{1}\left(<S_{0}\right)$ at the upper boundary $z=d$.

Following Shivakumara and Khalili [6], the governing linear stability equations in dimensionless form can then be written as

$$
\begin{aligned}
& {\left[\frac{\lambda}{P r} \sigma+B_{\mathrm{m}}|Q|+D a^{-1}\right]\left(D^{2}-a^{2}\right) W=-R_{\mathrm{t}} a^{2} \Theta+R_{\mathrm{s}} a^{2} \Sigma} \\
& {\left[D^{2}-a^{2}-Q D-\sigma\right] \Theta=f(z) W} \\
& {\left[\tau\left(D^{2}-a^{2}\right)-Q-\sigma \lambda\right] \Sigma=g(z) W}
\end{aligned}
$$

where $W, \Theta$ and $\Sigma$ are respectively the perturbed amplitudes of $z$-component of velocity, temperature and solute concentration, $D=\mathrm{d} / \mathrm{d} z$ is the differential operator and $a$ is the horizontal wavenumber. Here $R_{\mathrm{t}}=\alpha_{\mathrm{t}} g \Delta T d^{3} / v \kappa_{\mathrm{t}}$ is the thermal Rayleigh number, $D a=k / d^{2}$ is the Darcy number, $R_{\mathrm{s}}=\alpha_{\mathrm{s}} g \Delta S d^{3} / v \kappa_{\mathrm{t}}$ is the solute Rayleigh number, $Q=w_{0} d / \kappa_{\mathrm{t}}$ is the Peclet number, $\operatorname{Pr}=v \phi^{2} / \kappa_{\mathrm{t}}$ is the modified Prandtl number, $\lambda=\phi / A$ is the non-dimensional group, $\tau=\kappa_{\mathrm{S}} / \kappa_{\mathrm{t}}$ is the ratio of diffusivities, $B_{\mathrm{m}}=B_{\mathrm{d}} \phi^{2} / P r D a$ the non-dimensional group, $f(z)=-Q e^{Q z} /\left(e^{Q}-1\right)$ and $g(z)=-\tilde{Q} e^{\tilde{Q} z} /$ $\left(e^{\tilde{Q}}-1\right)$ are the dimensionless steady state non-linear basic temperature and solute concentration gradients respectively, $\tilde{Q}=Q / \tau$ and $\sigma$ is the growth rate. While $\phi$ is the porosity and $k$ is the permeability of the porous medium, $g$ is the gravitational acceleration, $A$ is the ratio of specific heats, $\kappa_{\mathrm{t}}$ is the thermal diffusivity, $\kappa_{\mathrm{s}}$ is the solute analog of $\kappa_{\mathrm{t}}, \alpha_{\mathrm{t}}$ is the volumetric expansion coefficient, $\alpha_{\mathrm{s}}$ is the solute analog of $\alpha_{\mathrm{t}}, B_{\mathrm{d}}=1.75 d_{\mathrm{g}} / 150(1-\phi)$ the form drag constant [7] and $d_{\mathrm{g}}$ is the diameter of the spheres.

The lower and upper boundaries of the porous layer are considered to be either impermeable (i.e. $W=0$ ) or porous (i. e. $D W=0$ ) but perfect conductors of heat (i. e. $\Theta=0$ ) and solute concentration (i. e. $\Sigma=0$ ). We note that the system of Eqs. (1)-(3) together with the boundary conditions constitutes a double eigenvalue problem. A single term Rayleigh-Ritz's method is used to solve the resulting eigenvalue problem which yields sufficiently accurate and useful results for the purpose in hand [2,8]. Accordingly, the variables in Eqs. (1)-(3) are written in terms of trial functions as $W=A W_{1}(z), \Theta=B \Theta_{1}(z)$, and $\Sigma=C \Sigma_{1}(z)$, where $W_{1}(z), \Theta_{1}(z)$, and $\Sigma_{1}(z)$ will be generally chosen in such a way that they satisfy the respective boundary conditions, and $A, B$ and $C$ are constants. Multiplying Eq. (1) by $W_{1}$, Eq. 
(2) by $\Theta_{1}$ and Eq. (3) by $\Sigma_{1}$; integrating the equations with respect to $z$ from 0 to 1 and eliminating $A, B$ and $C$ from the resulting equations, we get an expression for the eigenvalue $R_{\mathrm{t}}$ in the form (after omitting the subscript 1)

$$
R_{\mathrm{t}}=\frac{R_{\mathrm{s}}}{\tau} \frac{\Delta_{1}}{\Delta_{2}}+\left(\frac{\lambda}{P r} \sigma+B_{\mathrm{m}}|Q|+D a^{-1}\right) \frac{<(D W)^{2}+a^{2} W^{2}>}{a^{2}} \Delta_{1}
$$

where $\Delta_{1}=\frac{<(D \Theta)^{2}+\left(\sigma+a^{2}\right) \Theta^{2}+Q \Theta D \Theta>}{<W \Theta><-f(z) W \Theta>}, \Delta_{2}=\frac{<(D \Sigma)^{2}+\left(\sigma \lambda / \tau+a^{2}\right) \Sigma^{2}+\tilde{Q} \Sigma D \Sigma>}{<W \Sigma><-g(z) W \Sigma>}$ and $<\cdots>$ denotes the integration with respect to $z$ from 0 to 1 . The contributions arising due to throughflow may be distinguished clearly from the above equation. The terms with coefficients $Q$ and $\tilde{\mathrm{Q}}$ arise from the vertical transport of perturbation temperature and solute concentration respectively, while the terms involving $f(z)$ and $g(z)$ arise from the curvature of basic temperature and solute concentration respectively. Thus there will be an interaction of all these contributions on the eigenvalue and their effects have been determined for four different types of velocity boundary conditions, namely both boundaries impermeable $(I / I)$, both boundaries porous $(P / P)$, lower boundary impermeable and upper boundary porous $(P / I)$ and lower boundary porous and upper boundary impermeable $(I / P)$.

\subsection{Case (i): Both boundaries impermeable (I/I)}

The trial functions chosen are $W=z(1-z)=\Theta=\Sigma$ such that they satisfy the respective boundary conditions. Using these trial functions in Eq. (4) and performing the integration, we get

$$
R_{\mathrm{t}}=R_{\mathrm{s}}\left(\frac{\delta^{2}+\sigma}{\tau \delta^{2}+\lambda \sigma}\right) \delta_{1}+\left(\eta+\frac{\lambda}{\operatorname{Pr}} \sigma\right) \frac{\left(\delta^{2}+\sigma\right) \delta^{2}}{a^{2} \delta_{2}}
$$

where $\delta_{1}=\left[\frac{6 \tilde{Q} \operatorname{coth}(\tilde{Q} / 2)-\tilde{Q}^{2}-12}{6 Q \operatorname{coth}(Q / 2)-Q^{2}-12}\right] \tau^{4}, \delta_{2}=\frac{60}{Q^{4}}\left[Q^{2}-6 Q \operatorname{coth}(Q / 2)+12\right], \delta^{2}=10+a^{2}$ and $\eta=B_{\mathrm{m}}|Q|+D a^{-1}$.

It may be noted that $R_{\mathrm{t}}$ is an even function of $Q$ and hence the direction of throughflow does not change the value of $R_{\mathrm{t}}$. To examine the stability of the system, the real part of $\sigma$ is set to zero and take $\sigma=i \omega$ in Eq. (5) to get $R_{\mathrm{t}}$ in the form

$$
R_{\mathrm{t}}=R_{\mathrm{s}}\left(\frac{\tau \delta^{4}+\lambda \omega^{2}}{\tau^{2} \delta^{4}+\lambda^{2} \omega^{2}}\right) \delta_{1}+\left(\eta \delta^{2}-\frac{\lambda}{P r} \omega^{2}\right) \frac{\delta^{2}}{a^{2} \delta_{2}}+i \omega N
$$

where

$$
N=R_{\mathrm{s}}\left(\frac{\tau \delta^{2}-\lambda \delta^{2}}{\tau^{2} \delta^{2}+\lambda^{2} \omega^{2}}\right) \delta_{1}+\left(\eta+\frac{\lambda}{P r} \delta^{2}\right) \frac{\delta^{2}}{a^{2} \delta_{2}} .
$$

Since $R_{\mathrm{t}}$ is a physical quantity, it must be real and it implies either $\omega=0$ (stationary convection) or $N=0$ (oscillatory convection) in Eq. (6). The stationary convection occurs at $R_{\mathrm{t}}=R_{\mathrm{t}}^{s}$ which attains its minimum value at $a=\sqrt{10}$ and it is given by

$$
R_{\mathrm{t}, \min }^{\mathrm{s}}=\frac{R_{\mathrm{s}}}{\tau} \delta_{1}+\frac{40 \eta}{\delta_{2}} .
$$

When $N=0(\omega \neq 0)$, Eq. (7) yields a dispersion relation

$$
\omega^{2}=-\frac{\tau^{2} \delta^{4}}{\lambda^{2}}+R_{\mathrm{s}} \frac{a^{2}(\lambda-\tau) \delta_{1} \delta_{2}}{\lambda^{2}\left(\eta+\lambda \delta^{2} / P r\right)}=\frac{\tau P r a^{2} \delta_{2}}{\lambda\left(\eta P r+\lambda^{2}-\tau \delta^{2}\right)}\left(R_{\mathrm{t}}^{\mathrm{s}}-R_{\mathrm{t}}^{\mathrm{o}}\right) .
$$

For the occurrence of oscillatory convection, $\omega^{2}>0$ and this is possible only if

$$
\tau<\lambda \text { and } R_{\mathrm{s}}>R_{\mathrm{s}}^{*}=\frac{\tau^{2} \delta^{4}\left(\eta+\lambda \delta^{2} / \operatorname{Pr}\right)}{a^{2}(\lambda-\tau) \delta_{1} \delta_{2}} .
$$

Note that if $R_{\mathrm{S}}=0$ then $\omega^{2}<0$ and oscillatory convection does not occur. Further, if oscillatory convection occurs, it always takes place at a Rayleigh number lower than that of stationary convection. Substituting for $\omega^{2}$ 
from Eq. (9) into Eq. (6) with $N=0$, we get an expression for the Rayleigh number at which oscillatory convection sets in. That is,

$$
R_{\mathrm{t}}^{\mathrm{o}}=\left(\eta+\frac{\tau}{P r} \delta^{2}\right)\left[\frac{R_{\mathrm{s}}}{\lambda} \frac{\delta_{1}}{\left(\eta+\lambda \delta^{2} / P r\right)}+\left(\frac{\tau}{\lambda}+1\right) \frac{\delta^{4}}{a^{2} \delta_{2}}\right] .
$$

\subsection{Case (ii): Both boundaries porous (P/P)}

The trial functions considered are $W=1, \Theta=z^{2}-z=\Sigma$. As in the previous case, the conditions for the occurrence of stationary and oscillatory convection are obtained. The Rayleigh number for the stationary convection attains its minimum value at $a=0$ and it is given by

$$
R_{\mathrm{t}, \min }^{\mathrm{s}}=\frac{R_{\mathrm{s}}}{\tau} \beta_{1}+\frac{10 \eta}{\beta_{2}}
$$

where $\beta_{1}=\left[\frac{\tilde{Q} \operatorname{coth}(\tilde{Q} / 2)-2}{Q \operatorname{coth}(Q / 2)-2}\right] \tau^{2}$ and $\beta_{2}=\frac{5}{Q^{2}}[Q \operatorname{coth}(Q / 2)-2]$.

In the absence of an additional diffusing component and throughflow, we note that $R_{\mathrm{t}, \min }^{\mathrm{s}}=12 \mathrm{Da} \mathrm{a}^{-1}$ which is the known exact value [1]. Further, oscillatory convection occurs at

$$
R_{\mathrm{t}}=R_{\mathrm{t}}^{\mathrm{o}}=\left(\eta+\frac{\tau}{P r} \delta^{2}\right)\left[\frac{R_{\mathrm{s}}}{\lambda} \frac{\beta_{1}}{\left(\eta+\lambda \delta^{2} / P r\right)}+\left(\frac{\tau}{\lambda}+1\right) \frac{\delta^{2}}{\beta_{2}}\right]
$$

provided that

$$
\omega^{2}=\frac{\tau \operatorname{Pr} \delta^{2} \beta_{2}}{\lambda\left(\eta \operatorname{Pr}+\lambda \delta^{2}+\tau \delta^{2}\right)}\left(R_{\mathrm{t}}^{\mathrm{s}}-R_{\mathrm{t}}^{\mathrm{o}}\right)
$$

is positive. The necessary conditions for the occurrence of oscillatory convection are $\tau<\lambda$ and $R_{\mathrm{s}}>R_{\mathrm{s}}^{*}=\frac{\tau^{2} \delta^{2}\left(\eta+\lambda \delta^{2} / P r\right)}{(\lambda-\tau) \beta_{1} \beta_{2}}$.

\subsection{Case (iii): Lower boundary impermeable and upper boundary porous (P/I)}

The trial functions chosen for this case are $W=z^{2}-2 z$ and $\Theta=z^{2}-z=\Sigma$. It is found that the Rayleigh number for the occurrence of stationary convection attains its minimum value at $a=\sqrt{5}$ and is given by

$$
R_{\mathrm{t}, \min }^{\mathrm{s}}=\frac{R_{\mathrm{s}}}{\tau} \gamma_{1}+\frac{45 \eta}{\gamma_{2}}
$$

where $\gamma_{1}=\frac{\left(e^{Q}-1\right)\left[e^{\tilde{Q}}\left(\tilde{Q}^{3}-2 \tilde{Q}^{2}-6 \tilde{Q}+24\right)-2\left(2 \tilde{Q}^{2}+9 \tilde{Q}+12\right)\right]}{\left(e^{\tilde{Q}}-1\right)\left[e^{Q}\left(Q^{3}-2 Q^{2}-6 Q+24\right)-2\left(2 Q^{2}+9 Q+12\right)\right]}$.

and $\gamma_{2}=\frac{105\left[e^{Q}\left(Q^{3}-2 Q^{2}-6 Q+24\right)-2\left(2 Q^{2}+9 Q+12\right)\right]}{8 Q^{4}\left(e^{Q}-1\right)}$.

Unlike in the previous two cases, here we note that $R_{\mathrm{t}}^{\mathrm{s}}$ is not an even function of $Q$ and the direction of throughflow alters the stability of the system.

Further, oscillatory convection occurs at

$$
R_{\mathrm{t}}=R_{\mathrm{t}}^{\mathrm{o}}=\left(\eta+\frac{\tau}{P r} \delta^{2}\right)\left[\frac{R_{\mathrm{s}}}{\lambda} \frac{\gamma_{1}}{\left(\eta+\lambda \delta^{2} / P r\right)}+\left(\frac{\tau}{\lambda}+1\right) \frac{\left(5+2 a^{2}\right) \delta^{2}}{a^{2} \gamma_{1}}\right]
$$

provided that

$$
\omega^{2}=\frac{\tau P r a^{2} \delta^{2} \gamma_{2}}{\lambda\left(5+2 a^{2}\right)\left(\eta P r+\lambda \delta^{2}+\tau \delta^{2}\right)}\left(R_{\mathrm{t}}^{\mathrm{s}}-R_{\mathrm{t}}^{\mathrm{o}}\right)
$$

is positive. The necessary conditions for the existence of oscillatory convection are $\tau<\lambda$ and $R_{\mathrm{S}}>R_{\mathrm{S}}^{*}=\frac{\tau^{2} \delta^{2}\left(5+2 a^{2}\right)\left(\eta+\lambda \delta^{2} / P r\right)}{a^{2}(\lambda-\tau) \gamma_{1} \gamma_{2}}$. 


\subsection{Case (iv): Lower boundary porous and upper boundary impermeable (I/P)}

Since the transformations $z \rightarrow 1-z$ and $Q \rightarrow-Q$ leave the eigenvalue system of equations invariant, the results for this case can be deduced from the previous case by simply changing the sign of $Q$ in the expression for thermal Rayleigh number. This means that the results for upflow/downflow of the previous case correspond to that of downflow/upflow of the present case and vice versa.

\section{Results and discussion}

The effects of quadratic drag and throughflow on the onset of double diffusive convection in a porous layer are studied with the aim of understanding control of double diffusive convective instability by the adjustment of vertical throughflow. Fluid flow in the porous medium is described by the Forchheimer-extended Darcy equation. Four different types of velocity boundary conditions namely, $I / I, P / P, P / I$ and $I / P$ are considered for discussion. It is observed that the oscillatory convection occurs, irrespective of the boundary conditions, only when $\tau<\lambda$ and also the strength of solute concentration exceeds a threshold, which in turn depends on the nature of boundaries as well as on the strength and direction (in the case of only asymmetric boundaries) of throughflow.

Fig. 1(a) and (b) show the steady and oscillatory marginal stability curves in the $R_{\mathrm{t}}-a$ plane for both boundaries impermeable (symmetric boundaries), and for the lower boundary impermeable and upper boundary porous (asymmetric boundaries), respectively. The typical neutral curves shown here are for $\tau=0.01, B_{\mathrm{m}}=10, D a=0.003, \operatorname{Pr}=10$ and it may be noted that they are connected at only one bifurcation point. For the physical parameters considered, it is seen that oscillatory convection is preferred to stationary convection for both types of boundary conditions.

Fig. 2 depicts the stability boundaries drawn in the $R_{\mathrm{t}}^{\mathrm{c}}-R_{\mathrm{s}}$ plane for representative values of $Q=-0.5,0.5,5$, and $\lambda=0.5$ and 0.8 for different types of boundaries considered, where $R_{\mathrm{t}}^{\mathrm{c}}$ is the critical Rayleigh number (i.e., the least value among $R_{\mathrm{t}}^{\mathrm{s}}$ and $R_{\mathrm{t}}^{\mathrm{o}}$ with respect to the wavenumber). From the figure we note that $R_{\mathrm{t}}^{\mathrm{c}}$ is a piecewise linear function of $R_{\mathrm{s}}$ and the slope of $R_{\mathrm{t}}^{\mathrm{c}}-R_{\mathrm{s}}$ plot for $I / I$, $P / P$ and $P / I$ boundaries changes at

$$
\begin{aligned}
R_{\mathrm{s}}^{* *} & =\frac{Q^{4} \delta^{4}\left(\lambda \delta^{2}+\eta \operatorname{Pr}\right)}{60 a^{2} \tau^{2} \operatorname{Pr}\left[\tilde{Q}^{2}+12-6 \tilde{Q} \operatorname{coth}(\tilde{Q} / 2)\right](\lambda-\tau)} \\
R_{\mathrm{s}}^{* *} & =\frac{Q^{4} \delta^{4}\left(\lambda \delta^{2}+\eta \operatorname{Pr}\right)}{5 \operatorname{Pr}[Q \operatorname{coth}(Q / 2)-2](\lambda-\tau)}
\end{aligned}
$$

and

$$
R_{\mathrm{s}}^{* *}=\frac{8 Q^{4} \delta^{2}\left(5+2 a^{2}\right)\left(\lambda \delta^{2}+\eta P r\right)(e \tilde{Q}-1)}{105 a^{2} \tau^{2} \operatorname{Pr}\left[e^{\tilde{Q}}\left(\tilde{Q}^{3}-2 \tilde{Q}^{2}-6 \tilde{Q}+24\right)-2\left(2 \tilde{Q}^{2}+9 \tilde{Q}+12\right)\right](\lambda-\tau)}
$$

as does the preferred mode of instability. The value of $R_{\mathrm{S}}^{* *}$ at which the preferred type of instability occurs changes with the nature of boundaries and also depending on the values of physical parameters including the direction of throughflow in the case of

(a)

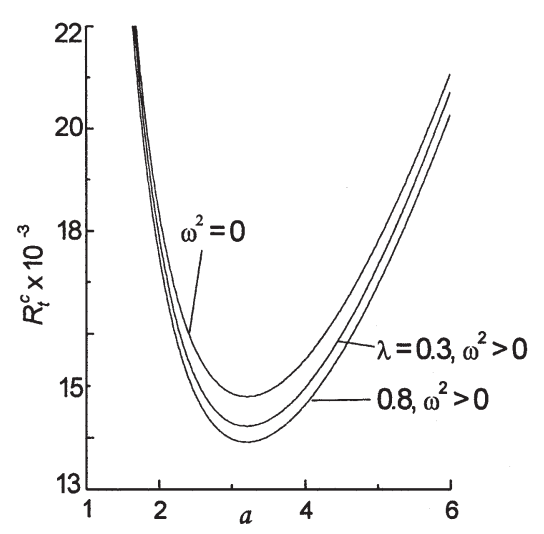

(b)

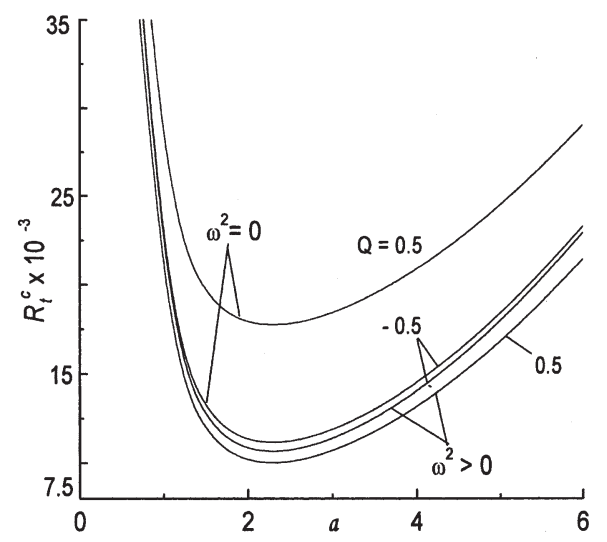

Fig. 1. Neutral stability curves for (a) $I / I$ boundaries when $|Q|=0.5$ and for (b) $P / I$ when $\lambda=0.8$ with $R_{\mathrm{s}}=500, \tau=0.01, B_{\mathrm{m}}=10, D a=0.003$ and $\operatorname{Pr}=10$. 


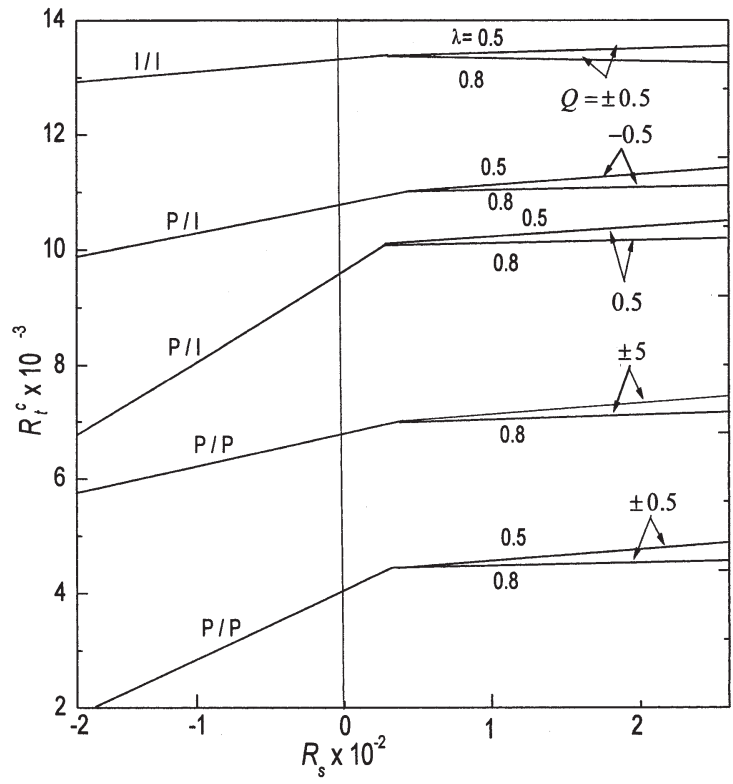

Fig. 2. Stability boundaries for $\tau=0.01, B_{\mathrm{m}}=10, D a=0.003$ and $P r=10$.

asymmetric boundaries. In this figure, the portion of each stability boundary lying to the left to the discontinuity in slope corresponds to steady onset, while to the right it is oscillatory onset. Further, it is evident that for a decrease in the value of $\lambda$ and also for the downflow (in the case of asymmetric boundaries) the region of stability increases and thus makes the system more stable.

In the case of a single component system (i.e., $R_{\mathrm{s}}=0$ ), it is well known that a small amount of throughflow in a particular direction destabilizes the system only when the boundaries are of different types [1-5]. In contrast to this, it is found that the critical Rayleigh number in the presence of an additional diffusing component ( i.e., $R_{\mathrm{s}} \neq 0$ ) manifests itself as minimum in the $R_{\mathrm{t}}^{\mathrm{c}}-Q$ plot irrespective of the nature of boundaries and the direction of throughflow. In other words, a small amount of throughflow in both the directions destabilizes the system initially and again makes the system stable with further increase in the value of $Q$ for all types of boundaries considered. The destabilization may be due to the distortion of steady state basic temperature and solute concentration distributions by the throughflow. A measure of these are given by the terms $\langle f(z) W \Theta\rangle$ and $\langle g(z) W \Sigma\rangle$. The values of $Q$ at which $R_{\mathrm{t}}^{\mathrm{c}}$ takes the least value in the $R_{\mathrm{t}}^{\mathrm{c}}-Q$ plot is denoted by $Q_{\mathrm{c}}$ and Table 1 represents these values for $I / I, P / P$ and $P / I$ boundaries for different values of $R_{\mathrm{s}}$ and $B_{\mathrm{m}}$ when $\tau=0.01, \operatorname{Pr}=10$ and $\lambda=0.8$.

Table 1

Critical Rayleigh and Peclet numbers for different values of $B_{\mathrm{m}}$ and $R_{\mathrm{S}}$ for different boundaries when $\tau=0.01, \operatorname{Pr}=10$ and $\lambda=0.8$

\begin{tabular}{|c|c|c|c|c|c|c|c|c|c|}
\hline \multirow[t]{2}{*}{$B_{\mathrm{m}}$} & \multirow[t]{2}{*}{$R_{\mathrm{s}}$} & \multicolumn{2}{|l|}{$I / I$} & \multicolumn{2}{|l|}{$P / P$} & \multicolumn{2}{|l|}{$P / I$} & \multicolumn{2}{|l|}{$P / I$} \\
\hline & & $R_{\mathrm{t}}^{\mathrm{c}}$ & $\left|Q_{\mathrm{c}}\right|$ & $R_{\mathrm{t}}^{\mathrm{c}}$ & $\left|Q_{\mathrm{c}}\right|$ & $R_{\mathrm{t}}^{\mathrm{c}}$ & $Q_{\mathrm{c}}$ & $R_{\mathrm{t}}^{\mathrm{c}}$ & $-Q_{\mathrm{c}}$ \\
\hline \multirow[t]{4}{*}{10} & 500 & 13,662 & 0.20461 & 4198 & 0.43252 & 9762 & 1.11174 & 10,141 & 0.13012 \\
\hline & 1000 & 13,715 & 0.26174 & 4268 & 0.58535 & 9805 & 1.19119 & 10,210 & 0.17191 \\
\hline & 2000 & 13,783 & 0.33037 & 4375 & 0.78180 & 9885 & 1.31285 & 10,297 & 0.22264 \\
\hline & 3000 & 13,834 & 0.37678 & 4462 & 0.92099 & 9957 & 1.40787 & 10,359 & 0.25759 \\
\hline \multirow[t]{4}{*}{15} & 500 & 13,701 & 0.18097 & 4222 & 0.37727 & 9900 & 0.83509 & 10,160 & 0.12300 \\
\hline & 1000 & 13,765 & 0.23484 & 4302 & 0.51958 & 9955 & 0.94192 & 10,235 & 0.16332 \\
\hline & 2000 & 13,847 & 0.29989 & 4421 & 0.70526 & 10,053 & 1.08871 & 10,330 & 0.21239 \\
\hline & 3000 & 13,907 & 0.34410 & 4516 & 0.83809 & 10,140 & 1.19628 & 10,397 & 0.24616 \\
\hline \multirow[t]{4}{*}{20} & 500 & 13,736 & 0.16382 & 4244 & 0.33694 & 10,003 & 0.62198 & 10,178 & 0.11686 \\
\hline & 1000 & 13,810 & 0.25103 & 4332 & 0.4698 & 10,075 & 0.75029 & 10,259 & 0.15604 \\
\hline & 2000 & 13,906 & 0.27697 & 4462 & 0.64526 & 10,194 & 0.91406 & 10,362 & 0.20359 \\
\hline & 3000 & 13,974 & 0.31924 & 4565 & 0.77189 & 10,296 & 1.02985 & 10,434 & 0.23627 \\
\hline
\end{tabular}


From the table the following conclusions can be drawn:

(i) Increase in the value of $R_{\mathrm{s}}$ and $B_{\mathrm{m}}$ is to delay the onset of convection.

(ii) The critical Rayleigh numbers are such that $\left[R_{\mathrm{t}}^{\mathrm{c}}\right]_{I / I}>\left[R_{\mathrm{t}}^{\mathrm{c}}(Q<0)\right]_{P / I}>\left[R_{\mathrm{t}}^{\mathrm{c}}(Q>0)\right]_{P / I}>\left[R_{\mathrm{t}}^{\mathrm{c}}\right]_{P / P}$ and thus indicating the system is more stable for impermeable boundaries, while it is more unstable for the porous boundaries.

(iii) Increase in $R_{\mathrm{s}}$ and decrease in $B_{\mathrm{m}}$ values is to increase the value of $Q_{\mathrm{c}}$. That means, the range of $Q$ up to which the system becomes destabilizing increases with an increase in $R_{\mathrm{s}}$ and decrease in $B_{\mathrm{m}}$. In particular, $\left[Q_{\mathrm{c}}(>0)\right]_{P / I}>\left[Q_{\mathrm{c}}\right]_{P / P}>\left[Q_{\mathrm{c}}\right]_{I / I}>\left[Q_{\mathrm{c}}\right.$ $(>0)]_{P / I}$.

(iv) In the case of asymmetric boundaries the direction of throughflow changes $R_{\mathrm{t}}^{\mathrm{c}}$ and the downward throughflow is found to be more stabilizing compared to the upward throughflow.

\section{Conclusion}

The presence of vertical throughflow is to deviate the otherwise linear basic temperature and solute concentration distributions to nonlinear ones which in turn affect the stability of the system significantly. The occurrence of oscillatory instability is found to be independent of the types of boundaries considered and it is a preferred mode of instability only when $\tau<\lambda$ and the solute Rayleigh number exceeds a threshold $R_{\mathrm{s}}^{*}$, which in turn depends on the nature of boundaries. The numerical calculations carried out reveal that, in contrast to the single component system, the throughflow destabilizes the system irrespective of the nature of boundaries of the porous layer. Further, the downward throughflow is seen to be more stabilizing than upward throughflow in the case of asymmetric boundaries. Thus it can be concluded that double diffusive convection in a porous layer may be controlled effectively by adjusting the rate of vertical throughflow.

\section{Acknowledgement}

The work reported in this paper has been supported by UGC under CAS programme. One of the authors (C.E.N.) wishes to thank the Management of Dr. Ambedkar Institute of Technology, Bangalore for its encouragement.

\section{References}

[1] D.A. Nield, A. Bejan, Convection in Porous Media, Second ed., Springer-Verlag, New York, 1999.

[2] D.A. Nield, Convective instability in porous media with throughflow, AIChE Journal 33 (1987) 1222-1224.

[3] I.S. Shivakumara, Boundary and inertia effects on convection in porous media with throughflow, Acta Mechanica 37 (1999) $151-165$.

[4] I.S. Shivakumara, C.E. Nanjundappa, Onset of convection in a sparsely packed porous layer with throughflow, Archives of Mechanics 53 (2001) $1-23$.

[5] A. Khalili, I.S. Shivakumara, Non-Darcian effects on the onset of convection in a porous layer with throughflow, Transport in Porous Media 53 (2003) 245-263.

[6] I.S. Shivakumara, A. Khalili, On the stability of double diffusive convection in a porous layer with throughflow, Acta Mechanica 152 (2001) $165-170$

[7] J.G. Georgiadis, J. Catton, Prandtl number effect on Benard convection in porous media, Transactions of ASME C: Journal of Heat Transfer 108 (1986) 284-290.

[8] C. Zhao, B.E. Hobbs, H.B. Muhlhaus, Theoretical and numerical analyses of convective instability in porous media with upward throughflow, International Journal for Numerical and Analytical Methods in Geomechanics 23 (1999) 629-646. 